\title{
Functional performance evaluation of an alginate/nano-cockle shell powder nanobiocomposite bone scaffold with BMP-2
}

\author{
NURNADIAH AHMAD ${ }^{1}$, HEMABARATHY BHARATHAM ${ }^{1, *}$ (, ZARIYANTEY ABDUL HAMID $^{1}$, \\ ENOCH KUMAR PERIMAL ${ }^{2}$ and PENNY GEORGE ${ }^{1}$ \\ ${ }^{1}$ Program of Biomedical Sciences, School of Diagnostic and Applied Health Sciences, Faculty of Health Sciences, \\ Universiti Kebangsaan Malaysia, Jalan Raja Muda Abdul Aziz, 50300 Kuala Lumpur, Malaysia \\ ${ }^{2}$ Physiology Unit, Department of Health Sciences, Faculty of Medicine and Health Sciences, Universiti Putra Malaysia, \\ Jalan UPM, 43400 Serdang, Selangor, Malaysia \\ *Author for correspondence (hema@ukm.edu.my)
}

MS received 19 August 2018; accepted 27 November 2018; published online 25 April 2019

\begin{abstract}
Improvement in bone tissue engineered scaffolds plays an important role in determining the healing outcome. This study aims to investigate the functional performance of previously developed nanobiocomposite bone scaffolds using bone morphogenetic protein-2 (BMP-2) for comparative analysis. The structural morphology and porosity of the scaffolds were analysed using scanning electron microscopy prior to dividing them into two groups, BMP-2 treated and control, for the surface mineralization study, in vitro and in vivo evaluation. The surface mineralization study showed the presence of plate-like calcium and phosphorus apatite crystallites in both groups indicative of the mineralization process. Osteoblasts (MC3T3-E1 subclone 4) cultured on the scaffold showed changes in the surface morphology with the presence of mineralization elements and the extensive presence of collagen fibres in BMP-2 scaffolds compared to the control. This was supported by a significant increase in calcium and alkaline phosphatase (ALP) activity showing enhancement in osteoconductiveness. In vivo studies conducted on 20 male ICR albino mice through subcutaneous dorsum implantation of scaffolds for 2 and 4 weeks showed a significant increase in the histological score for inflammatory cells, scaffold degradation and neovascularization at week 4 compared to week 2 for the BMP-2 scaffold. These findings are supported by a significant decrease in ALP activity indicative of scaffold mineralization. There were no significant differences between BMP-2 and control scaffolds within the period of this study. In conclusion, the functional performance of the scaffolds was mildly accelerated in BMP-2 compared to the control scaffold which showed promising properties in supporting osteogenesis without the use of any promoting factors.
\end{abstract}

Keywords. Nanobiocomposite bone scaffold; BMP-2; osteoblast; mineralization.

\section{Introduction}

Advancement in the field of biomaterials as materials for medicinal use has brought a concurrent expansion in the field of tissue engineering. Focus on fields such as bone tissue engineering has further narrowed down the use of biomaterials in forms of nanomaterials to increase the biological function to help in accelerating tissue healing and performance of the developed materials. In our previous study, we have successfully developed, characterized and evaluated a three-dimensional nanobiocomposite scaffold as a potential bone grafting material using nano-cockle shell powder from Anadara granosa sp. in combination with alginic acid polymers $[1,2]$. The pure aragonite form of calcium carbonate that makes up about 95 to $98 \%$ of cockle shells [3] is known to be analogous to the main mineral component of the bone, hydroxyapatite. In order to mimic the natural bone matrix structure, the study was undertaken using alginate, a naturally occurring polymer, as a representative of the organic component of the matrix. The polymer is known to be non-toxic, non-immunogenic, biodegradable and biocompatible [4] and has been regarded as safe by the Food and Drug Administration [5]. Bone morphogenetic protein2 (BMP-2) has shown potentials to induce mesenchymal stem cell differentiation into chondrogenic and osteogenic cells. Since its discovery, BMP-2 has been an important bone-inducing factor with osteoinductive properties that are extensively being studied for potential bone tissue engineering applications [6].

From initial studies, the nanobiocomposite bone scaffold in this relatively novel material combination has been shown to have sufficient morphological properties, pore size, porosity, degradability, mechanical properties and good biocompatibility [2]. In this study, we attempt to evaluate the functional performances of the scaffolds with regard to osteogenic responses by comparing between scaffolds 
loaded with BMP-2 and unloaded scaffolds as controls. We aim to highlight the potential use of the scaffold without any growth promoting factors with the intention of highlighting the material combinations used in the development of the scaffold in order to produce a cost-effective biomaterial based bone graft for future clinical applications.

\section{Materials and methods}

\subsection{Scaffold development}

Scaffolds were prepared according to the methods of Hemabarathy et al [2]. Briefly, pre-weighted nano-cockle shell powder was mixed with the prepared alginate hydrocolloid solution under constant stirring on a homogenizer stirrer machine at $600 \mathrm{rpm}$ until a homogenized smooth slurry is obtained. The scaffold mixture was prepared in a composition of alginate:nano-cockle shell powder of 40:60 (w/v). The completely homogenized mixture was poured into a custom-made cylindrical mold $(4 \mathrm{~cm}$ height $\times 1 \mathrm{~cm}$ diameter), labelled, allowed to set and frozen at $-20^{\circ} \mathrm{C}$ for $24 \mathrm{~h}$. The scaffolds were then transferred to a freeze dryer machine for lyophilization $\left(24 \mathrm{~h}\right.$ at $\left.-50^{\circ} \mathrm{C}\right)$. The lyophilized scaffolds were then removed from the molds and cross-linked with $1 \%$ calcium chloride solution for $20 \mathrm{~min}$ before further processing and re-lyophilization. The produced three-dimensional scaffolds are then divided into two groups consisting of control scaffolds and scaffolds that will be loaded with $5 \mu \mathrm{l}$ per scaffold of BMP-2 solutions $(0.1 \mathrm{mg}$ per $\mathrm{ml})$ before further studies.

\subsection{Scaffold surface mineralization}

Scaffold mineralization studies were performed according to the method of Whited et al [7] by submersion of scaffolds loaded with BMP-2 and control in $10 \times$ simulated body fluid (SBF). Both scaffolds were submerged in the $10 \times \mathrm{SBF}$ solution for $24 \mathrm{~h}, 7$ days and 14 days prior to microscopic evaluation. The scaffolds are first rinsed with copious amounts of deionized water to remove the salt residues before being fixed in $2.5 \%$ glutaraldehyde solution for $24 \mathrm{~h}$ for scanning electron microscopy (SEM) with energy dispersive X-ray (EDX) observation.

\subsection{In vitro studies for osteoconductiveness}

MC3T3-E1 subclone 4 pre-osteoblast cells at a density of 250,000 cells per scaffold were seeded onto scaffolds preadded with $5 \mu \mathrm{l}$ per scaffold of BMP- 2 solutions $(0.1 \mathrm{mg}$ per $\mathrm{ml}$ ) and $5 \mu \mathrm{l}$ per scaffold of $\alpha$-MEM media (control scaffolds). The cells were grown on the surface of the scaffolds for a period of 1, 7 and 14 days after which they were fixed in $2.5 \%$ glutaraldehyde for microscopic examination using a SEM. The intracellular ALP activity of the cells on the scaffolds was measured as an indicator of cell growth and proliferation according to Abdulahi and Zuki [3] using commercial kits (Abcam Inc., MA, USA).

\subsection{In vivo performance evaluation}

BMP-2 and control scaffolds were implanted subcutaneously on male ICR Albino mice (8-12 weeks of age) under general anaesthesia using IP Avertin (tribromoethanol). Prior to the

Table 1. Scaffold histology scoring (Cavallo et al [8]).

\begin{tabular}{|c|c|c|c|c|}
\hline Score & 0 & 1 & 2 & 3 \\
\hline Cellular infiltration & $\begin{array}{l}\text { Absence of cells in } \\
\text { contact with the } \\
\text { scaffold }\end{array}$ & $\begin{array}{l}\text { Cells observed at the } \\
\text { periphery but did not } \\
\text { infiltrate into the } \\
\text { scaffold }\end{array}$ & $\begin{array}{l}\text { Cells observed within } \\
\text { the periphery of the } \\
\text { scaffolds but not in } \\
\text { the centre }\end{array}$ & $\begin{array}{l}\text { Extensive penetration } \\
\text { of cells into the } \\
\text { scaffold }\end{array}$ \\
\hline $\begin{array}{l}\text { Collagenous bone } \\
\text { tissue formation }\end{array}$ & $\begin{array}{l}\text { Absence of } \\
\text { collagenous } \\
\text { matrix/dense } \\
\text { connective } \\
\text { tissues/bone tissues }\end{array}$ & $\begin{array}{l}\text { Few areas of early } \\
\text { collagen matrix } \\
\text { indicative of } \\
\text { connective tissue } \\
\text { formation }\end{array}$ & $\begin{array}{l}\text { Presence of dense } \\
\text { connective tissue } \\
\text { indicative of early } \\
\text { stage bone formation }\end{array}$ & $\begin{array}{l}\text { Presence of matured } \\
\text { bone tissues }\end{array}$ \\
\hline Scaffold degradation & $\begin{array}{l}\text { Original scaffold was } \\
\text { intact with clear } \\
\text { indication of its } \\
\text { margins }\end{array}$ & $\begin{array}{l}\text { Scaffolds partially } \\
\text { degraded, separated } \\
\text { by layers of cells and } \\
\text { connective tissues }\end{array}$ & $\begin{array}{l}\text { Scaffold extremely } \\
\text { degraded, difficult to } \\
\text { distinguish scaffold } \\
\text { from host tissue }\end{array}$ & $\begin{array}{l}\text { Scaffold completely } \\
\text { degraded, no } \\
\text { evidence of original } \\
\text { scaffold }\end{array}$ \\
\hline Fibrous encapsulation & $\begin{array}{l}\text { Extensive fibrous } \\
\text { encapsulation }\end{array}$ & $\begin{array}{l}\text { Moderate fibrous } \\
\text { encapsulation }\end{array}$ & $\begin{array}{l}\text { Mild fibrous } \\
\text { encapsulation }\end{array}$ & $\begin{array}{l}\text { No fibrous } \\
\text { encapsulation }\end{array}$ \\
\hline Neovascularization & $\begin{array}{l}\text { No blood vessels } \\
\text { observed }\end{array}$ & $\begin{array}{l}\text { Blood vessels observed } \\
\text { at the periphery of } \\
\text { the scaffold only }\end{array}$ & $\begin{array}{l}\text { Blood vessels } \\
\text { infiltrated the } \\
\text { scaffold but did not } \\
\text { reach its centre }\end{array}$ & $\begin{array}{l}\text { Blood vessels observed } \\
\text { in the centre of the } \\
\text { scaffold area }\end{array}$ \\
\hline
\end{tabular}




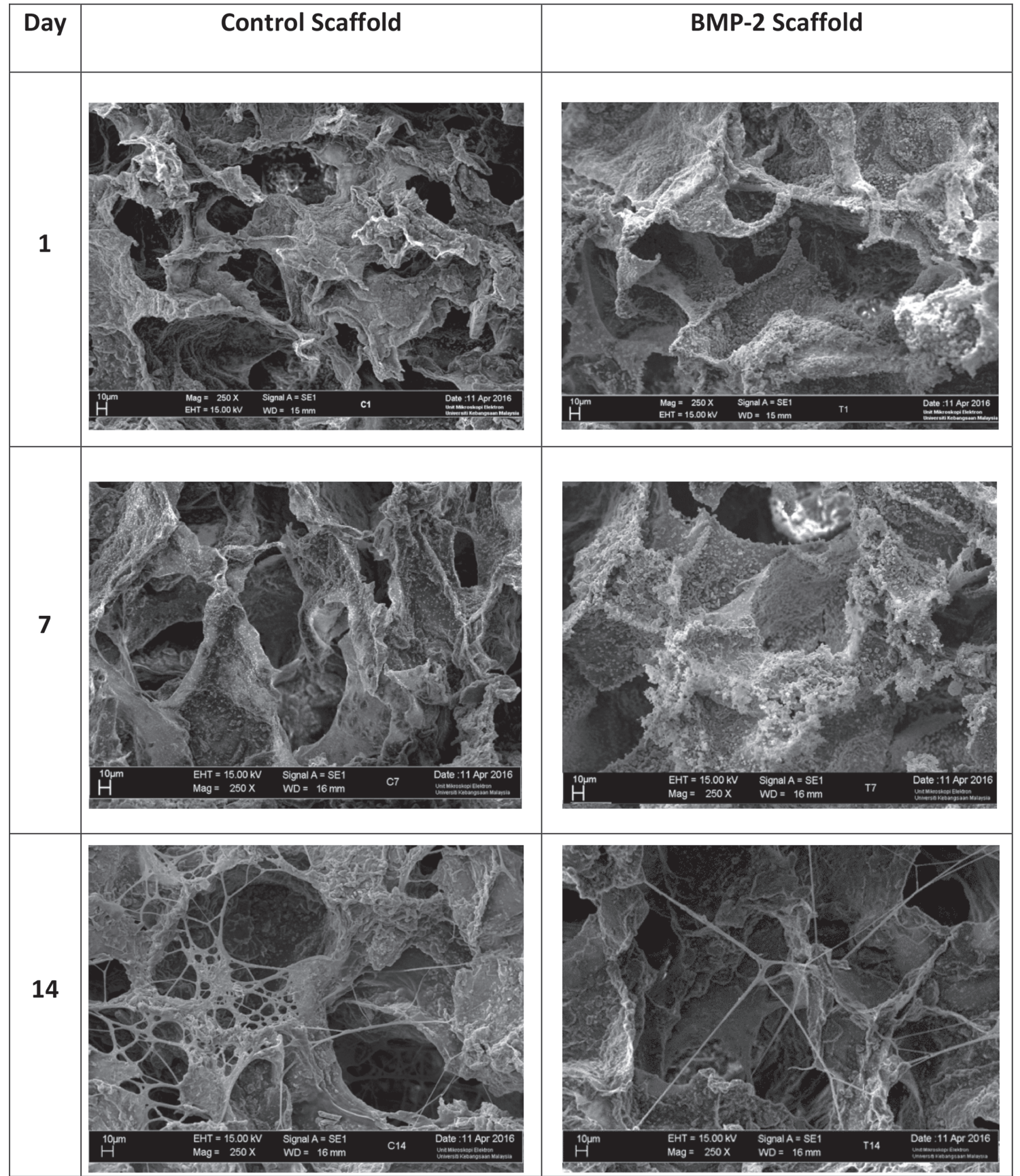

Figure 1. SEM micrograph of BMP-2 and control scaffold surfaces at days 1,7 and 14 of SBF immersion at $250 \times$ magnification.

surgical procedures, the animals were divided into two groups ( $n=10$ ) which represent weeks 2 and 4 of implantation duration. After the dorsal hairs were shaved, two incisions were made for the implantation of BMP-2 scaffolds (right dorsum) and the control scaffolds (left dorsum). The wounds were then closed with silk sutures and all animals were monitored daily. At weeks 2 and 4 of the study the scaffolds were harvested and cleaned for histology and biochemical analyses.

\subsection{Semi-quantitative histological scoring}

The harvested scaffold implants were fixed in $10 \%$ formalin and processed for Hematoxylin-Eosin (H\&E), Masson trichrome and von Kossa staining using standard protocols. The scoring criteria used for the evaluation of H\&E sections of the scaffolds were set based on the modified methods of Cavallo et al [8]. Scoring of the slides was carried out by 

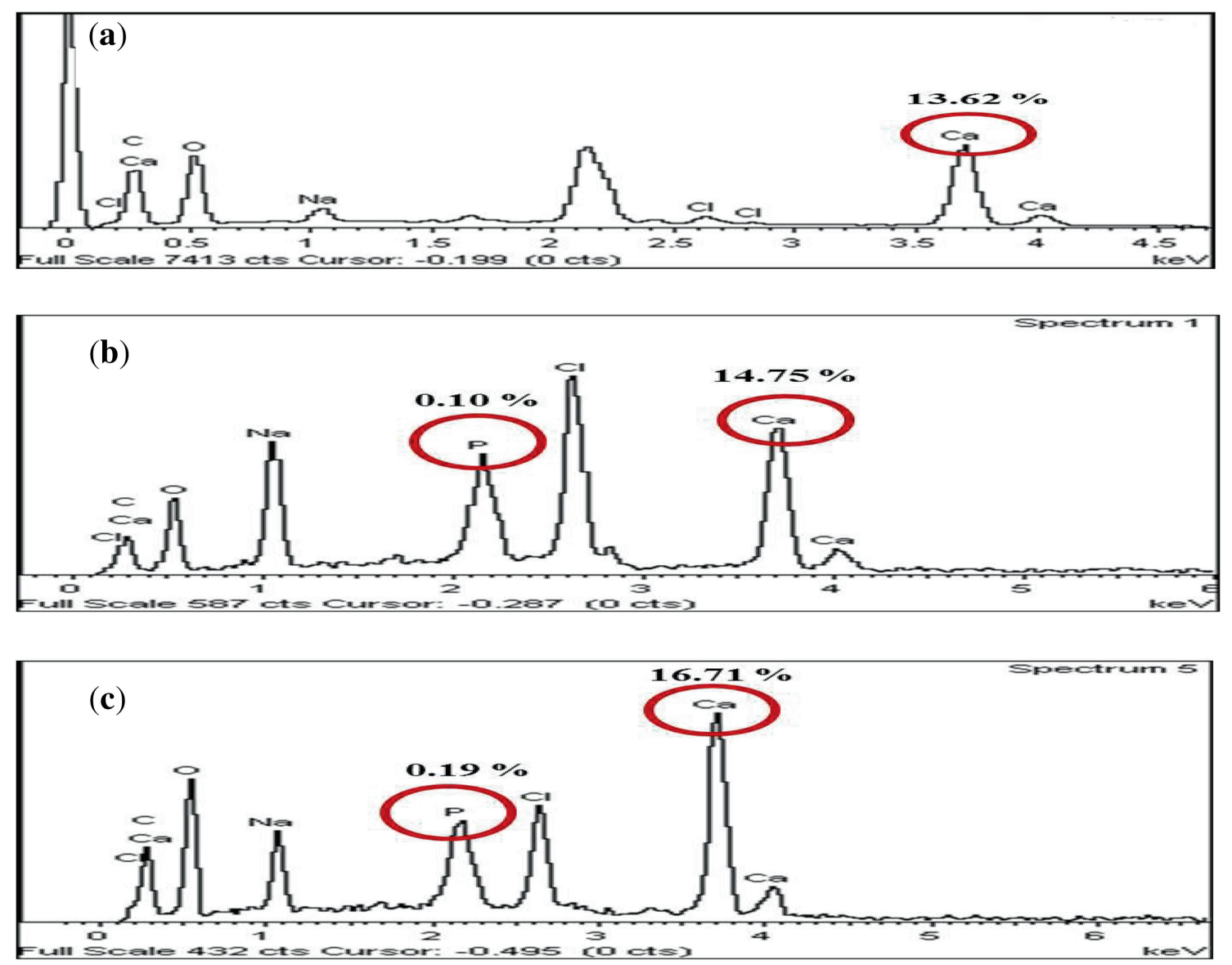

Figure 2. Elements detected in the fabricated scaffold without BMP-2 and prior to SBF immersion (a), control (b) and BMP-2 scaffolds (c) immersed in SBF. The result expressed as weight (\%).

blinded reviewers based on criteria stated in table 1 under $\times 4$ and $\times 10$ magnifications.

\subsection{Alkaline phosphatase (ALP) activity in scaffold post-implantation}

The ALP activity and calcium content were measured in scaffolds post-implantation as an indicator for osteogenic activity. The implants were harvested, frozen and crushed prior to being homogenized in lysis buffer $(0.2 \% \mathrm{NP}-40,10 \mathrm{mM}$ Tris $\mathrm{HCl}, 1 \mathrm{mM} \mathrm{MgCl}{ }_{2}, \mathrm{pH}$ 7.5). The sample (1 ml) was centrifuged at $12,000 \mathrm{~g}$ for $10 \mathrm{~min}$ at $4^{\circ} \mathrm{C}$ to obtain the supernatant that was assayed using the ALP and calcium kit by Abcam (Abcam Inc., MA, USA).

\section{Results}

\subsection{Scaffold surface mineralization}

Figure 1 shows the micrograph images of surface mineralization of BMP-2 and control scaffolds under $250 \times$ magnification on days 1, 7 and 14. Surface mineralization was evident as early as day 1 on both scaffolds with higher intensity observable in BMP-2 scaffolds. The extensive presence of plate-like crystals and collagen fibre formation were observable on both scaffolds by day 14 indicating the occurrence of the mineralization process. The evidence of this process could be further supported by the presence of calcium and phosphate elements detected through coupled EDX analysis as shown in figure 2 . The need for mineralization of a biomaterial with intended bone repair applications is necessary to support the formation of mechanically strong bonds with the existing bone tissue [9]. The formation of $\mathrm{CaP}$ phases on the scaffold matrix provides further indication of the fabricated scaffold ability to integrate with bone tissues [10] which were equally evident from both the BMP-2 and control scaffolds immersed in SBF.

\subsection{In vitro scaffold characterization studies}

Figure 3 shows the micrograph images of control and BMP-2 scaffold surfaces under $250 \times$ magnification. The attachment and proliferation of osteoblasts on the surface of scaffolds could be observed as early as day 1 . The mineralization of the scaffold matrices was evident by day 7 that were 


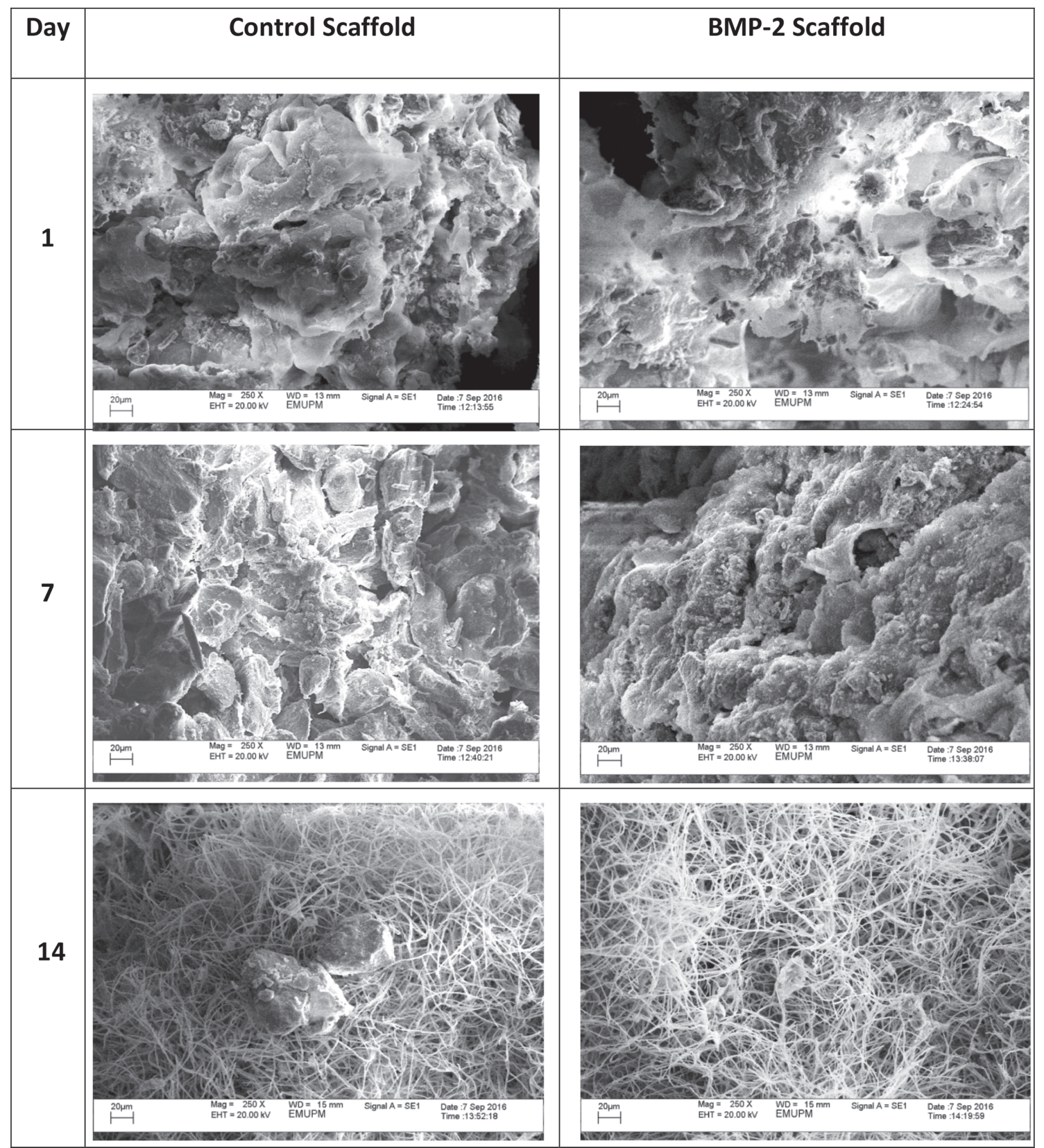

Figure 3. SEM micrograph of control and BMP-2 scaffolds under $250 \times$ magnification showing the growth and proliferation of osteoblasts on the surface at days 1,7 and 14 .

observable as apatite-like calcium phosphate mineral deposits which were found to be more intense on the BMP-2 scaffolds compared to control scaffolds. These findings are further supported by the detection of CaP elements through EDX analysis (figure 4) that indicated the mineralization of scaffold matrices by day 7 . The ability of a scaffold to facilitate cell attachment and matrix mineralization is a crucial aspect of assessing the effectiveness of the scaffold in facilitating new bone tissue formation $[11,12]$. The potential of the scaffolds was evident on day 14 , in which scaffolds from both groups showed an extensive presence of collagen fibres. The thread-like fibres produced by osteoblasts form the underlying organic structure in the process of bone formation. The maturation of the scaffold matrices was also evident through the expression of ALP enzymes, corresponding to the mineralization of the matrices and the synthesis of collagenous fibres as shown in figure 5 . A significant increase $(p<0.05)$ in ALP expression was noted on day 7 compared to day 1 in BMP-2 scaffolds and control scaffolds that significantly decreased by day 14 . The up-regulation and down-regulation of ALP expression were similar to those reported by Zuki et al and Wang et al $[13,14]$. The ALP enzyme is a transient marker known to be expressed 


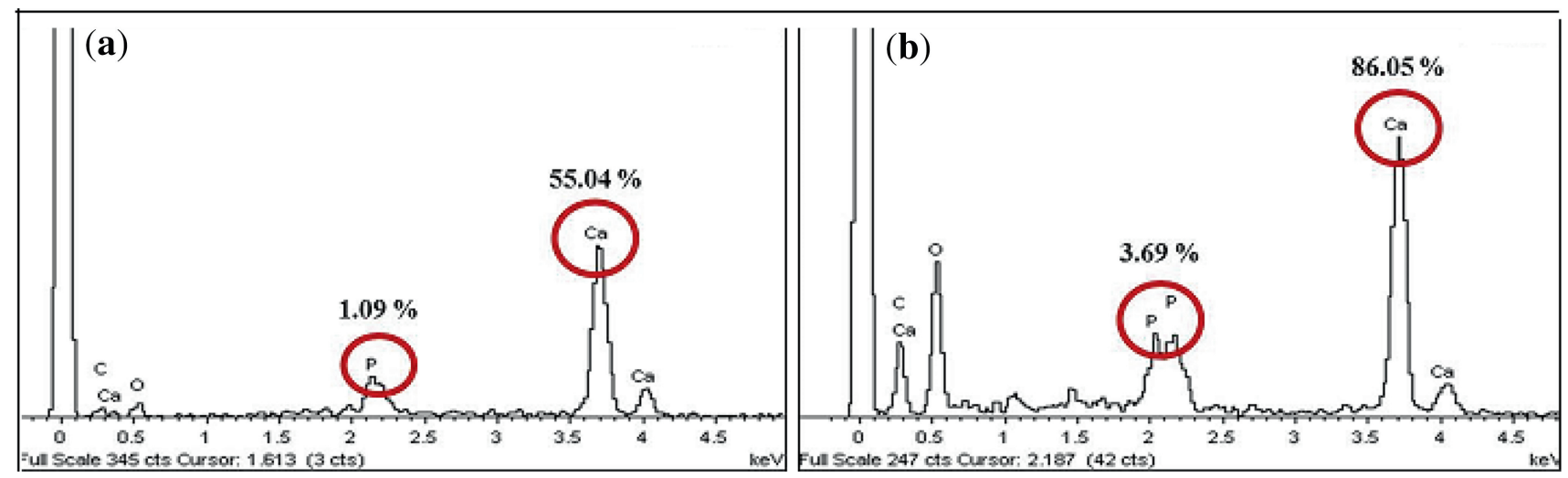

Figure 4. EDX graph showing the presence of calcium and phosphate detected on (a) control and (b) BMP-2 scaffolds. The result expressed as weight $(\%)$.

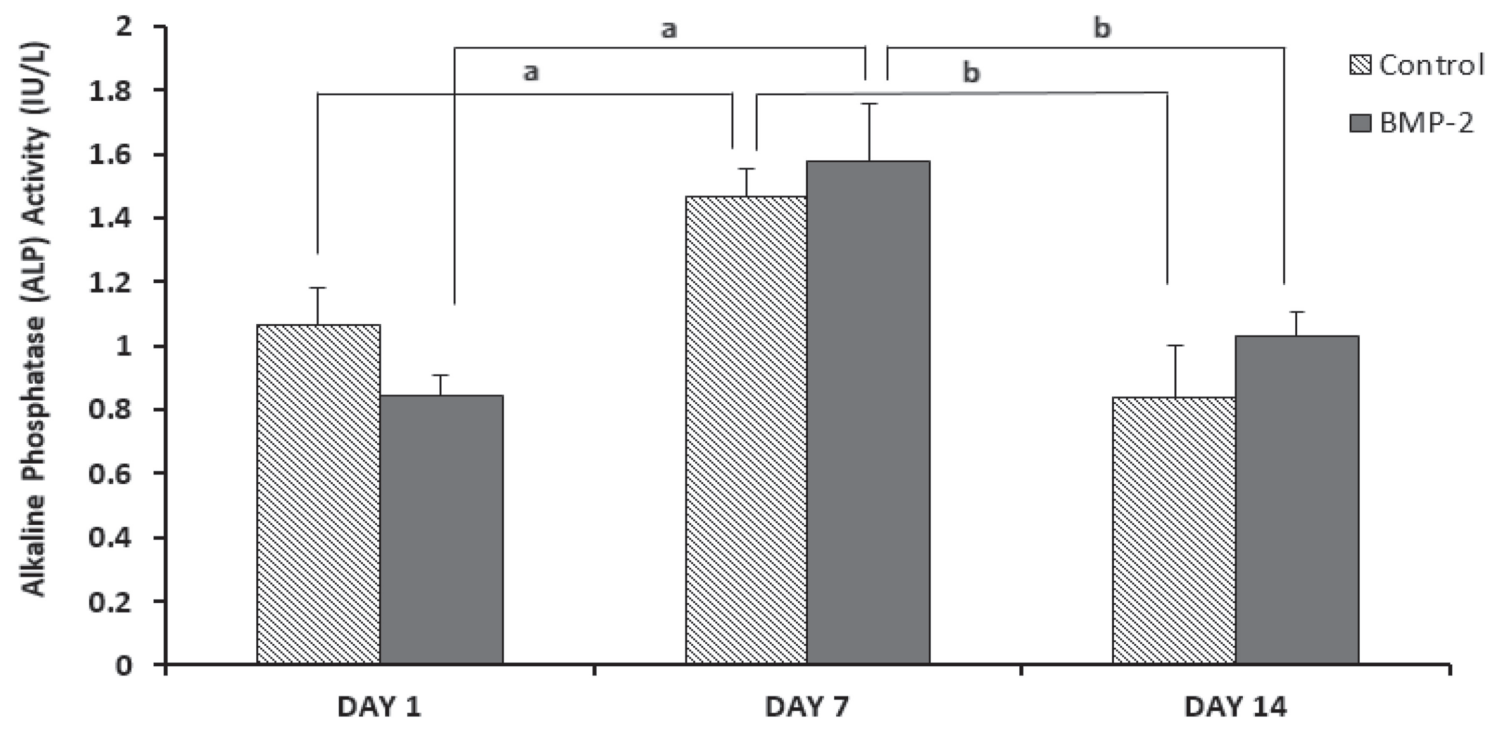

Figure 5. ALP activity on control and BMP-2 scaffolds at days 1,7 and 14 . Data present as mean \pm SEM. ${ }^{\mathrm{a}} p<0.05$ $v s$. day 1 and ${ }^{\mathrm{b}} p<0.05$ s. day 7 .

in early bone cell differentiation and is closely related to bone calcification in which a spike in the expression is often noted before mineralization of the bone matrix. These findings indicated that this scaffold supported the process of mineralization as early as day 7 . The presence of nanophase calcium carbonate from the cockle shells is a potent factor for the growth and proliferation of osteoblasts that help in accelerating the mineralization process of the scaffold. An important observation from the in-vitro studies was on the performance of the scaffolds that were almost equivalent by the end of the experimental time point. This highlighted the potential of the nanobiocomposite scaffold in facilitating bone formation without the need for additional growth factors.

\subsection{In vivo histological studies}

The subcutaneous scaffold implantation model is a widely accepted model for bone formation evaluation studies using
BMP-2 [15]. This model is commonly used for observing the initial tissue inflammatory response, biocompatibility, tissue vascularization and host recellurization of the extracellular matrix and is highly recommended for preclinical studies [16]. Figure 6 shows the scaffold section from control and BMP-2 scaffolds at weeks 2 and 4 at $\times 4$ magnification. Peripheral encapsulation was evident in both scaffolds and was found to be thicker at week 2. Although there was no statistical significance in scores, it was observable that the fibrous encapsulation decreased by week 4 (figure $6 \mathrm{c}$ and d). The formation of fibrous capsules is a crucial factor in limiting the prolonged inflammatory responses that could lead to implant rejection. An ideal scaffold would eventually show evidence of diminished capsulation with progress in time as an indication of limitation in the development of a chronic inflammatory response towards the implanted material [17]. Thinning of the fibrous capsule correlates with the increase in cellular infiltration in BMP-2 

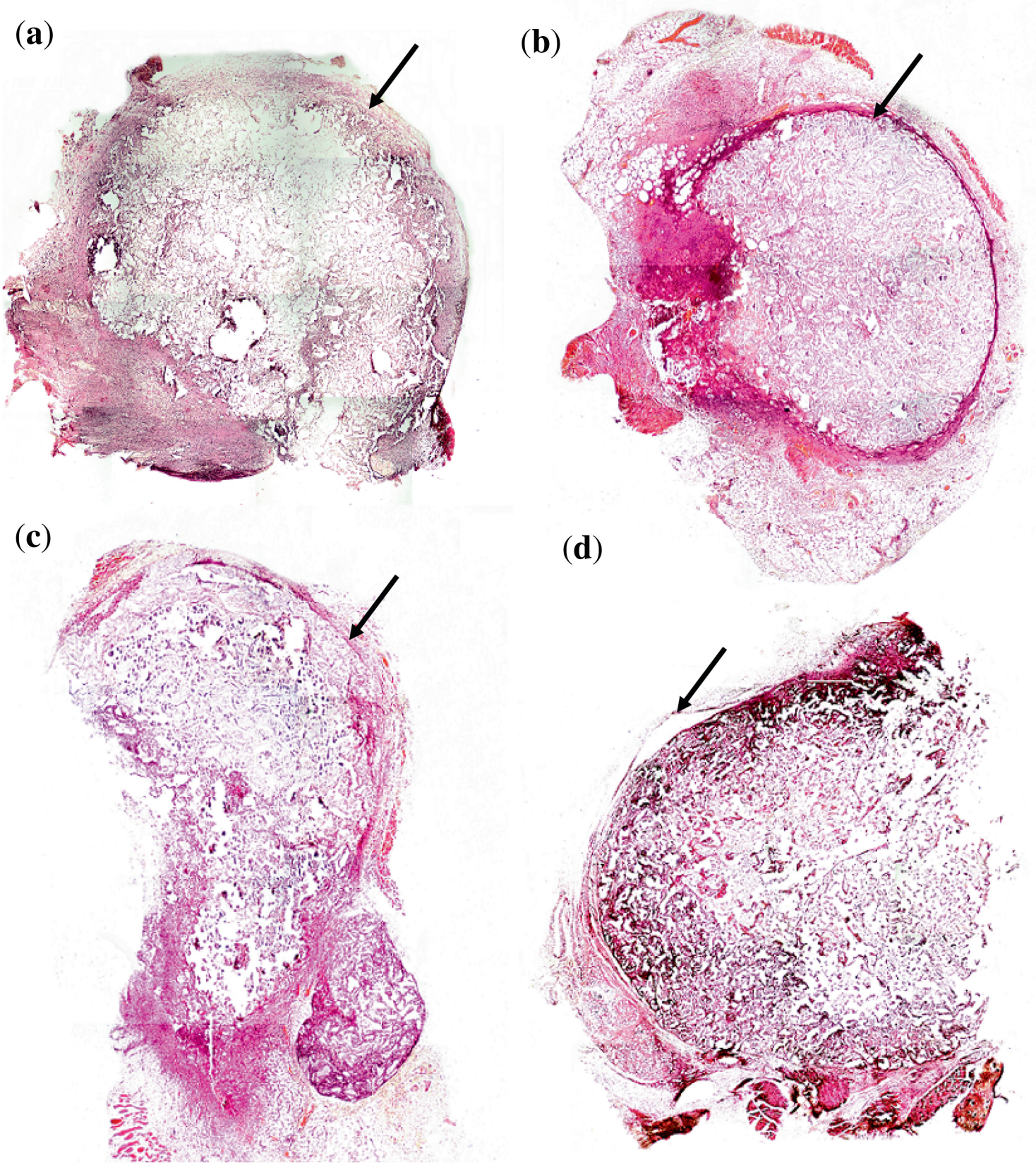

Figure 6. Scaffold sections from (a) control and (b) BMP-2 scaffolds at week 2 and (c) control and (d) BMP-2 scaffolds at week $4(\times 4$ magnification, $H \& E)$. Arrow indicates fibrous encapsulation.

scaffolds. These findings also correlated with the significant increase in the early formation of collagen tissues, scaffold degradation and new blood vessel formation in BMP-2 scaffolds compared to control scaffolds (figure 7). Corresponding to this, a higher rate of scaffold degradation was also scored at week 4 compared to week 2 in the BMP-2 scaffolds in relation to the increase in cellular activities, neovascularization and cellular infiltration. Figure 8 shows the section obtained from the centre of the scaffolds which clearly shows the presence of new blood vessels and increase in staining intensity indicating higher cellular infiltration in BMP-2 scaffolds at week
4. In the control scaffolds blood vessel formation was evident at the periphery margins. A significant increase in scores was also obtained for blood vessel formation in the BMP-2 scaffold at week 4 compared to week 2. Cellular infiltration was evident in scaffolds from both groups by week 2. Infiltrating cells were evident at the centre of the scaffold sections that scored higher in scaffolds from week 4 with significantly higher scores in BMP-2 scaffolds. Upon closer observation using Masson trichrome stains, as shown in figure 9, it was evident that the infiltrating cells were fibroblasts that were derived from mesenchymal cells from the skin. The fibroblasts 


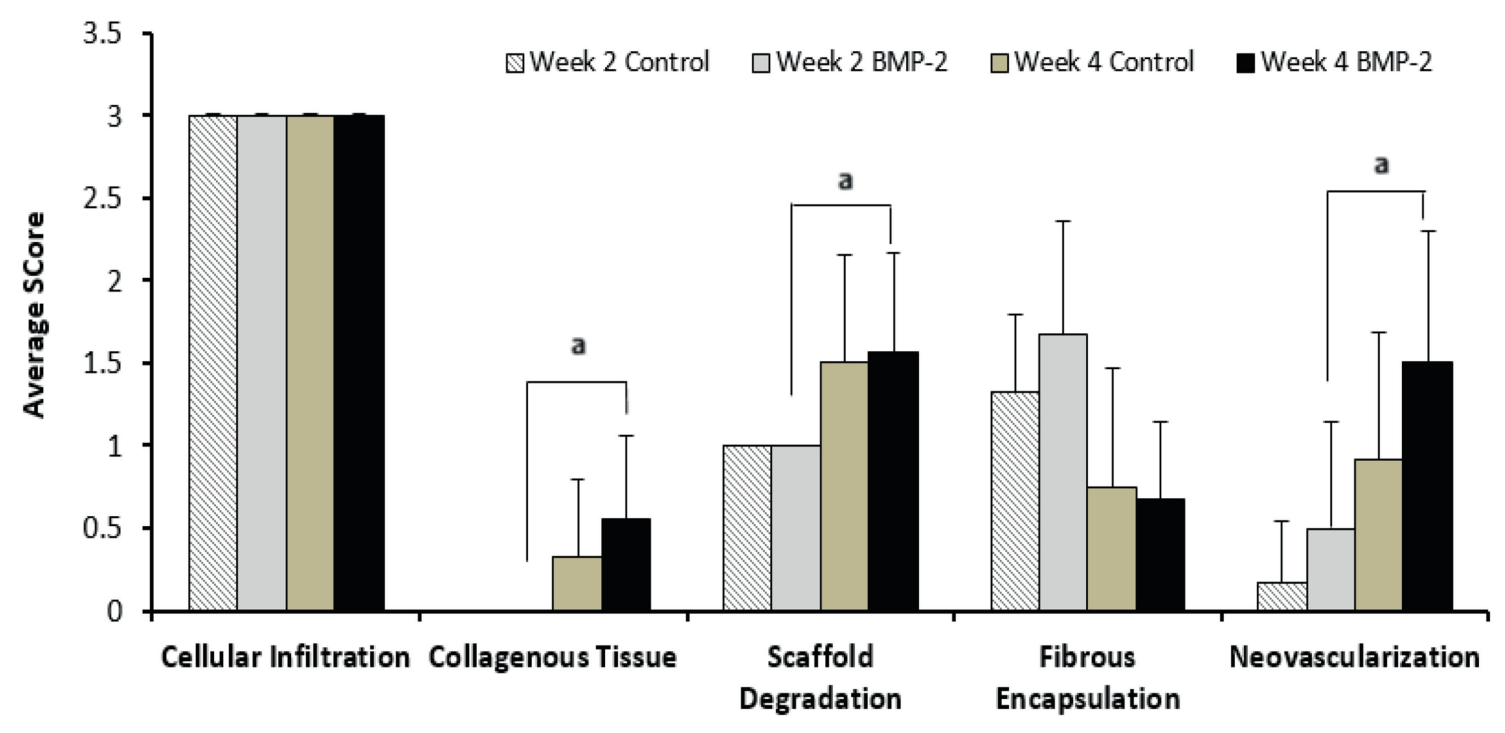

Figure 7. Average score obtained from histological scoring of control and BMP-2 scaffolds at weeks 2 and 4 $\left({ }^{\mathrm{a}} p<0.05\right.$ vs. week 2$)$.
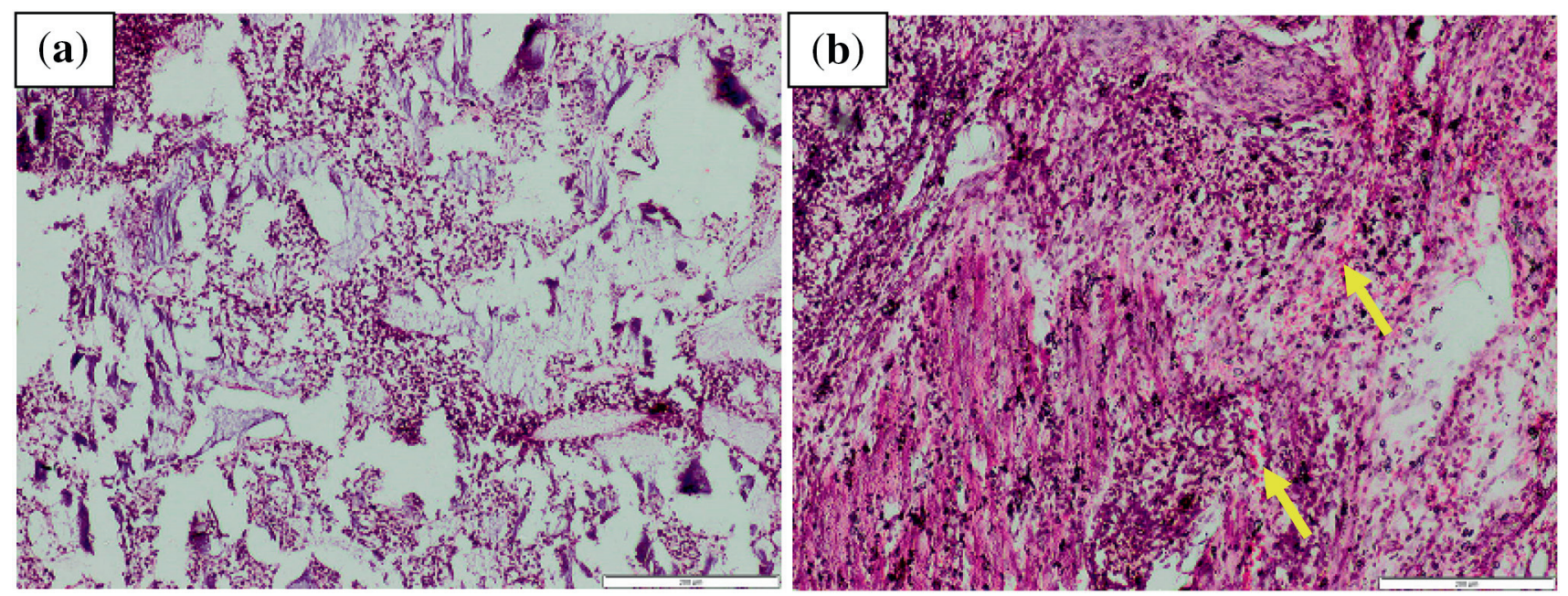

Figure 8. Section obtained from the centre of the scaffolds which clearly shows the presence of new blood vessels (arrow) and higher cellular infiltration in BMP-2 (b) compared to control scaffolds (a) at week 4 ( $\times 10$ magnification, H\&E).

were observed to have formed collagen tissues as an early indication of bone tissue formation. This could be supported by the presence of osteoid tissues and the mineralization process indicated by von Kossa stained sections as observed in figure 10. The high osteoconductive properties and the ability of the scaffolds to facilitate the collagen tissue formation and the presence of BMP-2 were noted to have accelerated this process. Though there was an increase in all the parameters scored for BMP-2 scaffolds, significant increases were only noted between week 4 as compared to week 2 within the BMP-2 scaffolds and not with the control. This further showed that the performance of the scaffold without BMP-2 was equally good but occurred at a slightly slower pace.

\subsection{ALP activity in scaffold post-implantation}

Figure 11 shows the significant decrease in ALP activity in both control and BMP-2 scaffolds at week 4 compared to week 2. These findings are in trend with the increased mineralization observed in the scaffolds through von Kossa stains at week 4 that clearly indicates an early stage bone formation in progress. In this study, we were unable to identify the presence of matured bone tissue or osteoblasts that are known factors for ALP expression. However, ALP enzymes that are known markers for developing bone cells are also expressed by fibroblasts $[18,19]$. Fibroblasts were the predominant cells that were observed through histological staining from both groups of scaffolds in this study. 

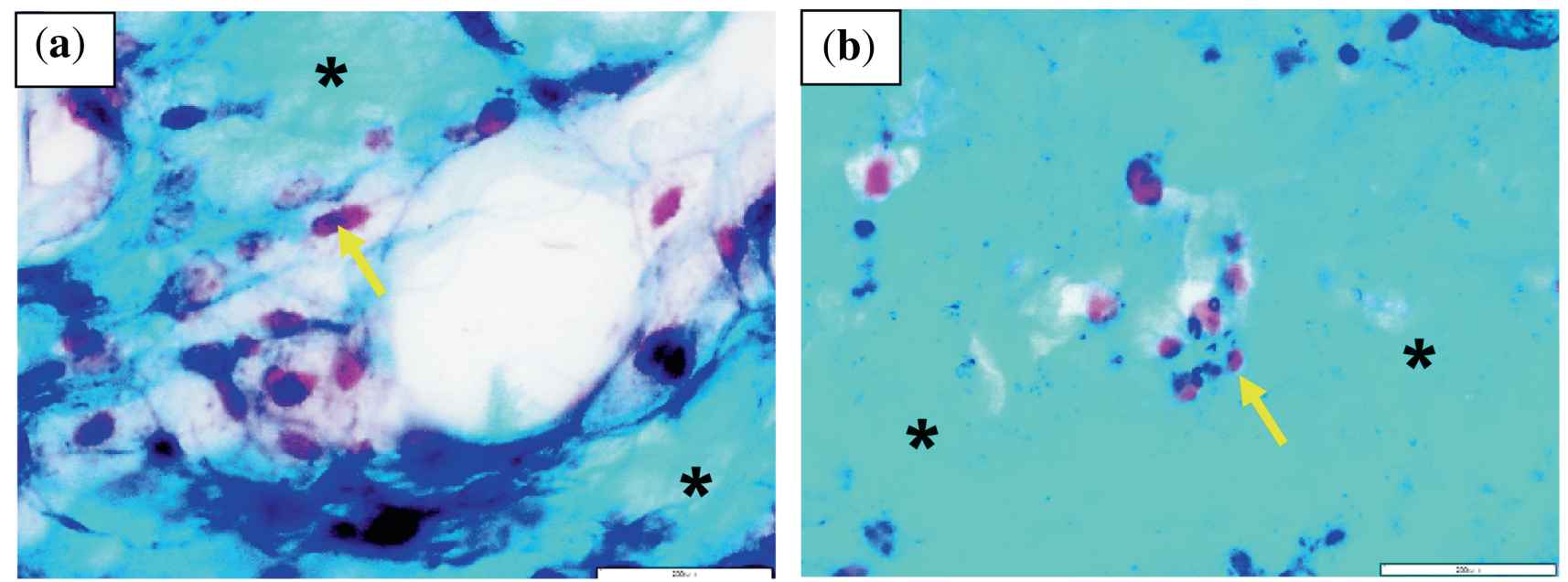

Figure 9. Masson trichrome stained scaffold sections clearly showing the presence of fibroblast nucleus (arrow) and dense collagen tissues (*) in (a) control and (b) BMP-2 scaffolds at week 4 ( $\times 10$ magnification).
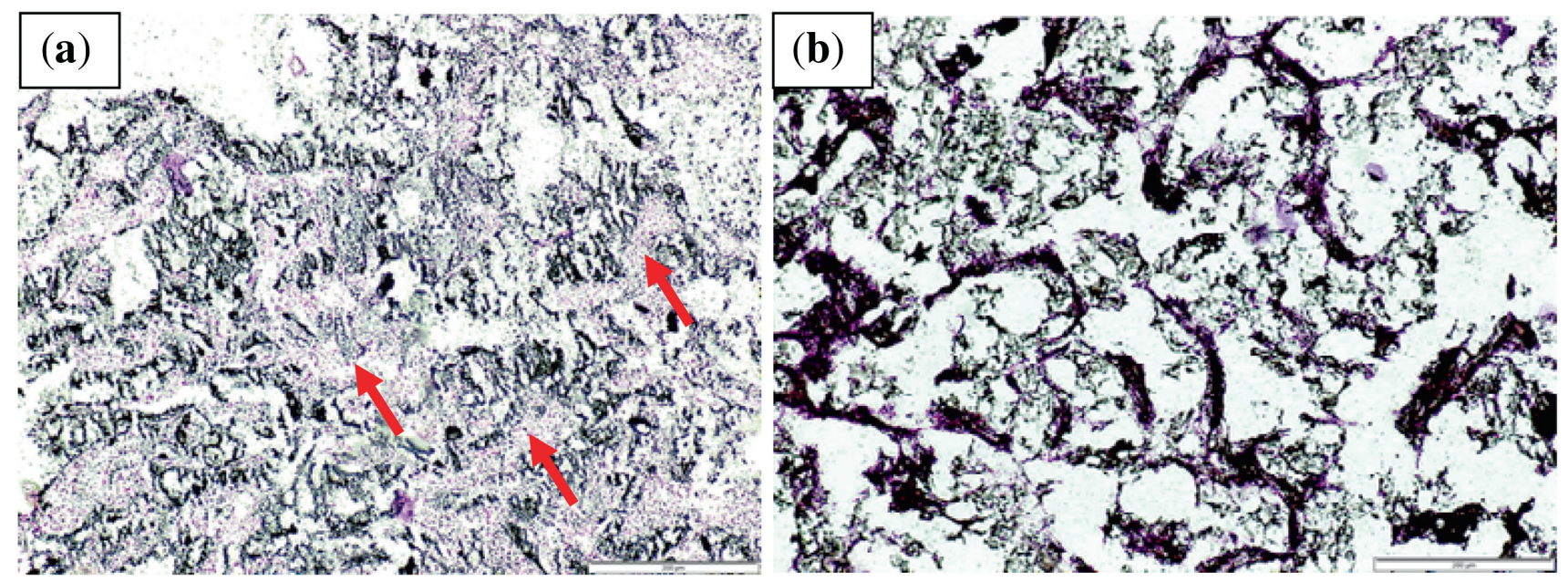

Figure 10. von Kossa stained scaffold section showing osteoid tissues (arrow) and the presence of the mineralization process (black deposits) in (a) control and (b) BMP-2 scaffolds at week 4 ( $\times 10$ magnification).

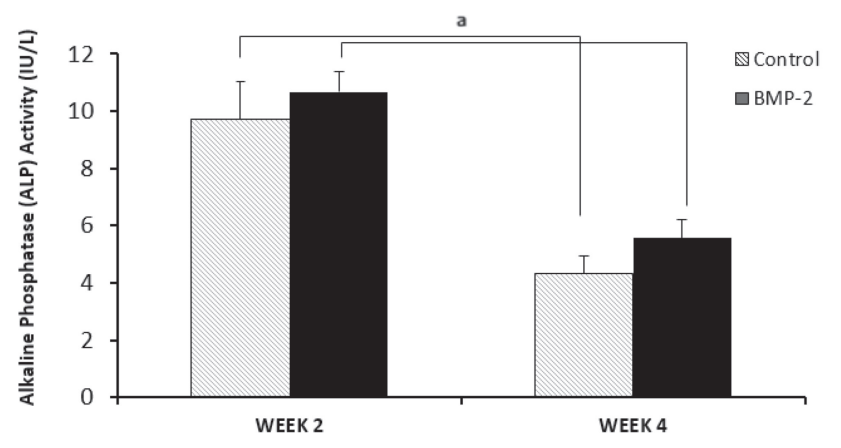

Figure 11. ALP activity on BMP-2 and control scaffold at weeks 2 and 4. Data present as mean \pm SEM. ${ }^{\mathrm{a}} p<0.05$ vs. week 2 .

Fibroblasts are known to be chemotactic towards specific growth factors [20] and have been shown to play a crucial role in bone re-growth. The presence of BMP-2 in the scaffolds could have a chemotactic effect which leads to the increase in cellular infiltration. The ALP expression by fibroblasts was previously noted in a study by Fathima and Harish [18] in which the increase in ALP expression from fibroblasts grown on the tricalcium phosphate bone graft material indicated a bone stimulatory effect. Similar stimulatory effects can be observed in this study in which the expression of ALP was found to be decreasing as mineralization progressed by week 4.

\section{Conclusions}

The findings of our study showed that the comparative performance of the scaffold showed only a slight enhancement when loaded with BMP-2 and in the absence of the growth 
factor. Although there was a slight improvement in BMP-2 scaffolds, these enhancing effects that were expected to occur did not show any conclusive results comparatively. The control scaffolds performed equally well indicating that the fabricated nanobiocomposite scaffold has promising applications for bone regeneration even without the use of performance-enhancing factors. The presence of the nanophase calcium carbonate in the fabricated scaffold produced ultra-fine structures on the scaffold matrix that provides a larger surface area for higher reactivity as well as with a stronger bonding property which further improves the overall performance of the scaffold. However, we do not deny that additional growth factors can be incorporated successfully with the scaffold to improve its application aspects further. We suggest that the concentration of BMP-2 used in this study could be cautiously increased to produce a significantly accelerated cellular response without overexpression. The enhancement of the scaffold performance with structural and biochemical modification as well as the growth factor carrier is currently being undertaken to explore the further possibilities of developing a fourth generation biomaterial based bone grafting material that is highly cost effective.

\section{Acknowledgements}

We thank the Electron Microscopy Unit of Universiti Kebangsaan Malaysia for the microscopy work and Universiti Putra Malaysia for providing the raw materials for the development of the scaffolds. This work was supported by Research University Grants (GGPM 2014-038).

\section{References}

[1] Hemabarathy B, Zuki A B, Enoch K P, Loqman M Y and Muhajir H 2014 Biomed. Res. Int. 672311

[2] Hemabarathy B, Zuki A B, Enoch K P, Loqman M Y and Muhajir H 2014 Sains Malays. 431023

[3] Abdullah S and Zuki A B 2014 Biomed. Res. Int. 50979

[4] Mooney D J and Lee Y K 2012 Prog. Polym. Sci. 37106

[5] Wong T M 2011 J. Pharm. Pharmacol. 631497

[6] Yoon B S and Lyons K M 2004 J. Cell Biochem. 9393

[7] Whited B M, Whitney J R, Hofman M C, Xu Y and Rylander M N 2011 Biomaterials 322294

[8] Cavallo J A, Roma A A, Jasielec M S, Ousley J, Creamer J, Pichert M D et al 2015 Ann. Surg. 261405

[9] Kepa K, Coleman R and Grondahl L 2015 Biosurf. Biotribol. 1214

[10] Kokubo T and Takadama H 2006 Biomaterials 27 2907

[11] Tampieri A, Sandri M, Landi E, Celotti G, Roveri N, Mattioli B M et al 2005 Acta Biomater. 1343

[12] Putnam A J and Mooney D J 1996 Nat. Med. 2824

[13] Zuki A B, Bahaa F H and Noordin M 2011 Regen. Med. Tissue Eng. (IntechOpen) 365

[14] Wang H, Li Y, Zuo Y, Li J, Ma S and Cheng L 2007 Biomaterials 283338

[15] Shi Q, Li Y, Sun J, Zhang H, Chen L, Chen B et al 2012 Biomaterials 336644

[16] Reza K, Jason L G, Christopher N, Souman J, Eva M, Amir L et al 2018 Acta Histochem. 120282

[17] Goreish H H, Lewis A L, Rose S and Lioyd A W 2004 J. Biomed. Mater. Res. A 68

[18] Fathima H and Harish 2015 J. Nat. Sci. Biol. Med. 6291

[19] Yamaguchi M, Shimizu N, Shibata Y and Abiko Y 1996 J. Dent. Res. 75889

[20] Nishimura F and Terranova V P 1996 J. Dent. Res. 75986 